\title{
Cultura Cultura
}

\section{La confrontation des trois premières catégories de la Logique de la philosophie d'Eric Weil avec la dialectique hégélienne}

Confronto entre as três primeiras categorias da Logique de la philosophie de Eric Weil e a dialéctica hegeliana

\section{Michel Renaud}

\section{OpenEdition}

\section{Journals}

\section{Edição electrónica}

URL: https://journals.openedition.org/cultura/1790

DOI: $10.4000 /$ cultura. 1790

ISSN: 2183-2021

Editora

CHAM - Centro de Humanidades

Edição impressa

Data de publição: 1 dezembro 2013

Paginação: 63-69

ISSN: 0870-4546

Refêrencia eletrónica

Michel Renaud, «La confrontation des trois premières catégories de la Logique de la philosophie d’Eric Weil avec la dialectique hégélienne », Cultura [Online], Vol. 31 | 2013, posto online no dia 25 novembro 2014, consultado o 21 setembro 2021. URL: http://journals.openedition.org/cultura/1790 ; DOl: https://doi.org/10.4000/cultura.1790

Este documento foi criado de forma automática no dia 21 setembro 2021.

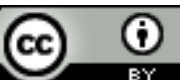

Cultura is licensed under a Creative Commons Atribuição 4.0 International. 


\title{
La confrontation des trois premières catégories de la Logique de la philosophie d'Eric Weil avec la dialectique hégélienne
}

\author{
Confronto entre as três primeiras categorias da Logique de la philosophie de \\ Eric Weil e a dialéctica hegeliana
}

Michel Renaud

1 Un vrai philosophe a ceci de particulier que nous ne cessons jamais d'apprendre avec lui. Plus nous le relisons, plus il nous enrichit du sens qui se dégage de son discours. Ce sens, nous le recevons sur l'horizon de toutes nos lectures et de tout ce qui, peu à peu et selon les vicissitudes de notre existence, a façonné notre réceptivité ainsi que notre capacité d'interprétation. Ce que le texte nous donne à comprendre est également le fruit d'une projection nôtre, lourde de toutes nos expériences vécues et de ces idées plus ou moins conscientes qui forment le tissu de nos présupposés. Eric Weil est ainsi l'un de ces philosophes que nous avons tout intérêt à relire, car il ne cesse de nous surprendre, au double sens du terme; il nous étonne par la puissance de sa pensée et nous réserve la surprise d'une découverte toujours renouvelée à la mesure même de nos relectures. Aujourd'hui, cette surprise coïncide avec la reprise de sa logique et les reprises auxquelles elle nous invite.

2 Le propos de cet article est très limité; il consiste à relire le début de la Logique de la philosophie et de le confronter à la pensée de Hegel. La conclusion devrait se déplacer vers la fin de la Logique, pour vérifier si la superposition de la catégorie finale et de la première catégorie se réalise sans hiatus ; c'est en fin de compte la question de la circularité de la Logique qui se profile à l'arrière-plan du concept de reprise, comme s'il s'agissait de la «reprise» totale de l'œuvre. Initialement programmée, cette seconde partie de notre communication sera seulement ébauchée à titre de conclusion. 
Il est sans doute banal de répéter que la Logique de la philosophie apparaît comme une des tentatives les plus réussies de refaire pour notre temps une Logique qui évoque le style d'une Phénoménologie de l'esprit, en tenant compte de l'évolution de la pensée après Hegel. Du reste, la référence à Hegel est explicite; mais elle ne suffit pas à éclaircir par ellemême ce qui relie ou délie Eric Weil de Hegel. Pour ma part, je ne puis échapper à une question extrêmement simple; alors que le Hegel de l'Encyclopédie des sciences philosophiques commence sa Logique par la dialectique de l'être, Eric Weil privilégie la catégorie de la vérité. Quel est le sens de cette différence et que gagne-t-on à cet échange, quels sont ses présupposés ? Je ne cacherai pas l'aspect exploratoire de cette intuition; il est possible que ce parallélisme ne résiste que faiblement à l'analyse ; il vaut néanmoins la peine de le suivre en son moment initial, avec l'idée que même son échec éventuel ne manquerait pas d'être instructif.

\section{L'être et la vérité.}

Dans la petite Logique de Hegel, au § 86, l'être est le concept dans toute son immédiateté ; l'absence de médiation qui le caractérise le constitue en commencement «indéterminé, simple ». C'est déjà la totalité en tant que non déployée et se maintenant dans l'élément de la pensée pure. On pourrait dire, ajoute Hegel, que le point de départ est Dieu, mais ce ne serait possible que si Dieu était l'être même et ce choix ajouterait une médiation à l'être ; mais le commencement pur ne peut être lui-même médiatisé. L'être est donc la catégorie la plus pauvre, car elle se présente comme vide de tout contenu, bien qu'elle soit en même temps virtuellement riche de tous les contenus auxquels son développement donnera vie.

Chez Weil, on le sait, le moment initial est la vérité. «Nous avons commencé par une attitude, celle de la Vérité, l'attitude la plus pauvre du point de vue logique, celle de la satisfaction dans la présence totale, tellement totale qu'il n'y a pas de discours, même pas de langage, que, en apparence au moins, il n'y existe pas encore de catégorie pour l'homme de cette attitude, mais seulement pour le philosophe qui se tourne vers elle » (p. 86). D'un côté, notons que Hegel mentionne la possibilité de commencer également par le Vrai absolu, mais celui-ci contient encore une médiation en plus de l'être; de l'autre, Weil indique que la vérité implique le concept de totalité, qui nous semble correspondre au terme «absolu » présent chez Hegel. Comme celui-ci, Weil montre que la "vérité est tout ", mais que ceci est déjà un langage qui explique la vérité, tandis que l'attitude dans sa pureté, ne souffre aucune médiation explicative. Le choix de la Vérité se justifie par sa relation au discours, précisément parce que la philosophie est avant tout discours. Et l'auteur d'en être conscient : c'est la vérité qui « apparaît comme première sur le plan des attitudes qui mènent à la quête de la cohérence et du contentement, ne serait-ce que parce que c'est elle qui s'annonce dans l'éternelle nostalgie du contentement et de la présence qui caractérise tous les discours de l'homme»(p. 86). Dès la fin de 1 'introduction, le ton est donné; en opposition à la violence, l'être humain cherche le contentement dans la présence. Le ton est plus anthropologique que chez Hegel, mais par ailleurs, le traitement que Weil réserve au discours l'oriente vers une ontologie des catégories. ${ }^{1}$

6 Il importe toutefois de souligner que la pauvreté initiale de l'être chez Hegel conditionne le rythme dialectique de toute la philosophie. C'est probablement ici que Hegel se distingue le plus de la métaphysique scolastique, pour laquelle l'être est l'acte d'exister, 
au sens le plus riche de l'expression. De la même façon, c'est parce que la vérité, selon Weil, est la catégorie la plus pauvre qu'elle peut mettre en marche le mouvement dialectique.

7 A la fin du premier chapitre sur la Vérité, Weil s'explique largement sur le choix de la Vérité (p. 93-94) : «Cependant l'histoire de la réflexion a montré que les deux termes d'Etre et de Dieu sont plus difficiles à manier, si l'on veut éviter les impasses de l'ontologie de la réflexion qui oppose sujet et objet, et de la foi qui, à moins de faire de l'ontologie sous le titre d'ontologie, s'arrête à la négation de tout concret et refuse et la doctrine et la réflexion ». Weil considère ainsi que mettre l'être au départ de la philosophie ne parvient pas à éviter la confusion entre l'être et l'objet (p. 94); c'est pourquoi il discorde de Hegel: "l'Etre qui forme le point de départ de sa logique n'est pas, et il évite ainsi les diffi cultés insolubles de la réflexion de l'Etre dans le sujet ou du sujet dans l'Etre (...) » (p. 93). En fin de compte, le choix de la Vérité se justifie pour des raisons de nature "pédagogique ", si dans la tradition occidentale, l'être est assimilé à l'objet.

8 Il est tout de même étrange que ce soit pour des raisons semblables que Hegel et Weil, respectivement, critiquent la solution alternative: pour Hegel le vrai inclut déjà une médiation qui est réflexion, ce qui l'empêche d'être un pur commencement, tandis que pour Weil, l'être est soit, en tant que non réfléchi, un arrêt qui bloque toute progression, soit, en tant que réfléchi, une dissociation en sujet et objet, ce qui l'empêche d'être originaire. Il n'empêche que le motif qui nous paraît essentiel pour Weil est le contexte du discours, qui privilégie la catégorie de la vérité. En d'autres termes, ce sont les présupposés d'avant la dialectique qui justifient le point de départ.

\section{Le néant et le non-sens.}

9 Le parallélisme de Hegel et Weil est plus suggestif dans l'approche de la deuxième catégorie, celle du néant hegelien et du nonsens de Weil. L'introduction du néant (\$87) advient par le fait que «cet être pur est l'abstraction pure, partant l'absolument-négatif, qui, pris pareillement en son immédiateté, est le néant ». L'on n'a pas encore atteint le niveau de la réflexion, car l'être et le néant qui en est la conclusion se maintiennent, dirait-on avec Hegel, dans l'extériorité de leur immédiateté. Le néant qui sera passé par le moment de la réflexion au-dedans de soi sera chez Hegel l'essence, qui inaugure la seconde partie de la Science de la logique. Au niveau du néant, toute réflexion intérieure est encore absente. Cependant, toutes les formes ultérieures de mouvement impliquent l'altérité inhérente à la négativité propre au néant.

10 Chez Eric Weil, c'est le non-sens qui occupe cette fonction. Les exemples peuvent aider à la compréhension; Parménide d'abord, dans la mesure où tout ce qui n'est pas l'un est sans rapport avec lui ; si l'un est la vérité qui n'a pas même besoin de se dire pour être telle, ce qui n'est pas l'un est donc non-sens. Il est significatif que l'exemple du bouddhisme soit présent aussi bien chez Hegel, dans son commentaire au paragraphe du néant (\$ 87) que chez Weil. « Pour le bouddhisme - nous dit celui-ci -, la vie n'a pas de sens et elle n'a pas de vérité » (p. 96). Notons que le néant de la vérité n'est pas le faux, mais le non-sens. Tout comme chez Hegel l'être n'est rien, de même, chez Weil, « le nonsens et la vérité ne se distinguent pas : il faut reconnaître la Vérité comme le non-sens, le non-sens comme la Vérité. Et cette reconnaissance elle-même est non-sens. Elle n'est que le préambule, le programme qui doit être réalisé » (p. 96). L'idée de programme montre à 
sa façon que la découverte du non-sens est, en tant qu'altérité de la vérité pure, ce qui est destiné à penser les êtres et la subjectivité en leur vérité propre.

11 Le concept de reprise apparait alors pour la première fois, tout au moins dans la Logique; ${ }^{2}$ il est défini comme «la compréhension d'une attitude (ou catégorie) nouvelle sous une catégorie précédente, compréhension réalisée dans et par cette attitude antérieure » (p. 98). Arrêtons-nous un instant sur cette définition fondamentale. Il semble bien en effet qu'elle exprime un aspect du mouvement dialectique, mais en un sens inverse du dépassement ou de la "sursomption ", c'est-à-dire de l'Aufhebung hegelienne. Chez ce dernier, l'Aufhebung est la production, sur la base des deux moments dialectiques antérieurs, d'une forme nouvelle, irréductible aux moments dont elle émerge. Ainsi, l' Aufhebung exprime la double négation à la faveur de laquelle surgit une attitude ou forme nouvelle. Chez Weil, la reprise est l'interprétation d'une attitude sur la base du langage spécifique d'une ou de toutes les catégories antérieures. Si je ne me trompe, ce mouvement de réinterprétation n'est pas thématisé de cette façon par Hegel. Que gagnet-on alors avec la reprise de Weil ? On y légitime la possibilité d'une interprétation en miroir, mais rétrospective, où une attitude vécue et non encore comprise se trouve exprimée en un langage qui ne lui convient pas encore adéquatement, en tant qu'il s'agit du langage spécifique de la catégorie antérieure. Le fait que Weil recoure au concept kantien de schéma (p. 82) pour exprimer la fonction de la reprise indique la nouveauté de ce processus dialectique. Nous assistons, grâce au concept de reprise, à un enveloppement progressif des figures les unes dans les autres, mais cet enveloppement permet l'apparition d'une nouveauté qui ne se dégage pas de manière absolument nécessaire à partir des figures, attitudes ou catégories antérieures. La proximité de Weil avec Hegel est ainsi compatible avec une forme subtile de distanciation, que précisément le concept de reprise indique. En d'autres termes, le progrès de la dialectique hegelienne, qui se fait en vertu d'un développement nécessaire ${ }^{3}$ cède la place au projet de cohérence du discours, qui ne paraît pas redevable du même degré de nécessité dialectique. Certes, cette nécessité est plus visible lors de la présentation des premières catégories de Weil, mais les chapitres qui succèdent à la catégorie de l'absolu montrent qu'il s'agit d'une cohérence qui ne coïncide plus exactement avec la nécessité pure de type hegelien.

\section{Le vrai et le faux face au devenir.}

Le dépassement dialectique de l'être et du néant engendre le concept du devenir, en tant qu'à la fois, il est et il n'est pas (encore) ce qu'il est. Cette idée hegelienne est reprise dans la troisième catégorie de Weil, "le vrai et le faux». Selon ce dernier, "la Vérité, déterminée pour nous par la catégorie du non-sens, devient maintenant réelle pour ellemême. Elle devient le lieu de ce qui peut être dit ». La vérité, en elle-même était encore muette, parce que totalement indéterminée ; mais passant par la médiation du non-sens, elle entre pour elle dans l'univers de l'altérité, qui est le langage (p. 101).

13 Il est significatif que l'explication que Weil présente tout de suite dans le paragraphe suivant s'énonce en termes purement hegeliens. « Voilà tout ce qu'on peut dire : l'Etre est l'Etre, l'Etre est le néant, le néant est l'Etre. C'est peu de chose, et néanmoins, il y a un langage : le néant est, et le néant est néant, et l'Etre devient le domaine où le néant qui est s'oppose et se marie à l'Etre qui est néant : ce qu'on appelle l'existence des choses (pour Hegel, le devenir) est né » (p. 101). Alors que le langage apparaît seulement comme concept dans le chapitre sur « "La certitude sensible » de la Phénoménologie de l'esprit, il 
est ici le lieu dans lequel subsistent - ou peuvent subsister - côte à côte le vrai et le faux. Mais il n'y a pas encore de médiation entre les deux.

Weil se donne alors la peine de brosser les traits de l'attitude qui correspond à cette catégorie et la trouve dans l'interprétation de la philosophie présocratique. Certes il y le langage qui dit le vrai et le faux. Mais c'est seulement pour nous que le vrai et le faux s'opposent, en une contradiction dialectique. De cette manière, les grands présocratiques, Xénophane, Parménide, Héraclite, enseignent la vérité ; mais comme tout ce que l'on dit particularise la vérité, l'enseignement ne peut s'identifier avec la vérité ; il est donc faux. Mais le langage qui dit le faux peut coexister avec la vérité, car la vérité totale dans laquelle on se trouve est muette. Le rapport du maitre au disciple constitue ainsi l'attitude qui sous-tend cette catégorie. Ce qu'il y a de plus spécial dans l'analyse de Weil est que l'attitude en question échappe à la logique de la contradiction. "Le vrai est la vérité, le faux est tout le reste. Cela ne veut pas dire que la contradiction ne soit pas sentie (...).Cela montre seulement que la contradiction n'est pas catégorie, qu'on ne pose pas de questions dans l'intention d'y répondre à l'aide de la contradiction, qu'on ne se sert pas de la contradiction pour découvrir le vrai ou le faux, mais qu'on a besoin du vrai et du faux pour établir la contradiction » (p. 105).

Deux remarques nous sont suggérées par le langage d'Eric Weil. En premier lieu, sa dialectique est très proche de celle de Hegel, là même où on l'attendrait le moins. En effet, Hegel détermine comme " devenir » le résultat du mouvement de l'être et du néant, mais il ne s'agit pas encore du devenir de quoi que ce soit. Pour penser les choses qui sont dans le devenir, on doit disposer do concept de devenir avant celui de la chose même. Maintenant, chez Weil, ce n'est pas la contradiction qui constitue l'opposition entre le discours vrai et le discours faux, mais c'est l'existence logiquement préalable du vrai et du faux qui pourra faire surgir le moment de la contradiction dans le langage. Par sa réflexion sur la relation du maitre et du disciple au sein de la philosophie présocratique, Weil illustre historiquement l'attitude ici décrite du vrai et du faux.

Mais le moment de la reprise nous réserve une surprise. Quel est le sens exact de la reprise? Ne pourrait-on pas la comprendre de deux façons symétriques et peut-être complémentaires? La définition citée ci-dessus nous dit qu'il s'agit de la compréhension d'une catégorie nouvelle sous une attitude antérieure, « compréhension réalisée dans et par cette attitude antérieure » (p. 98). Mais il est possible d'entendre cette définition dans les deux sens opposés : d'un côté, la reprise fait comprendre la présence d'une catégorie antérieure au sein d'une attitude nouvelle, de telle sorte que l'attitude nouvelle en devient plus intelligible; le but est alors de montrer la richesse de la catégorie nouvelle qui en arrive à se comprendre mieux elle-même par la médiation de l'antérieure. Mais, par ailleurs, on pourrait énoncer la même relation en sens inverse : c'est la catégorie ancienne qui se comprend mieux par le fait de passer par le prisme de l'attitude nouvelle.

Or, la situation à laquelle fait allusion la reprise dans le chapitre sur le vrai et le faux est complexe ; elle montre d'abord que la non-médiation entre le vrai et le faux se trouve à la racine de nombreuses doctrines absolutistes, qui paraissent plus évoluées, plus riches en contenu, « reprennent les catégories dont elles se servent sous la simple unité bipolaire du vrai et du faux dès qu'elles s'adressent au "sentiment", à l"'évidence", à la "conviction intime" » (p. 106) ; mais elles ne sont pas pour autant de véritables reprises du vrai et du faux, "par le simple fait d'avoir un contenu déterminé, ce qui est impensable dans la catégorie du vrai, pour laquelle tout contenu déterminé est du côté du 
faux ». Cette simple observation montre déjà, dès le début de la Logique, la complexité du concept de reprise.

\section{NOTAS}

1. Eric Weil note en effet: "Pour l'individu, la réflexion précède la doctrine. Mais la réflexion n'est que dans la doctrine, qui est première. L'individu, philosophe ou non, ne se comprend pas lui-même. Il est compris (dans les deux sens du mot) dans la doctrine » (p. 92).

2. Cfr p. 97-99; 118-119; p. 81-82; 390-391. «La reprise, pour employer un concept kantien, est le schéma qui rend la catégorie applicable à la réalité et qui permet ainsi de réaliser concrètement l'unité de la philosophie et de l'histoire» (p. 82).

3. Cfr le commentaire de Hegel au $\S 87$, qui souligne le caractère nécessaire du développement: «(...) la pensée logique, moyennant laquelle de telles déterminations se produisent au jour non pas du tout d'une manière contingente, mais d'une manière nécessaire " (Encyclopédie des sciences philosophiques. T. 1. La science de la logique (trad. B. Bougeois), Paris, Vrin, 1970, p. 350.

\section{RESUMOS}

Este breve artigo relê o início da Lógica da Filosofia de Eric Weil e compara-o com o pensamento de Hegel. Para o efeito, confrontam-se as três primeiras categorias da Lógica da Filosofia e o início da primeira parte da "pequena Lógica" de Hegel, isto é, a secção Ser da Enciclopédia das Ciências Filosóficas. Tentar compreender aquilo que coloca o pensamento dialéctico em movimento, revela-se assaz interessante para o propósito: no caso de Weil, a Verdade; no de Hegel, o Ser. A mesma questão será colocada na análise da segunda categoria de Weil, Não sentido, cujo parentesco com o "nada" (das Nichts) em Hegel merece ser aferido. Surgirá, então, o conceito de "retomada" cujo contraste com a Aufhebung hegeliana se tornará notório. Finalmente, a categoria o Verdadeiro e o Falso permitirá precisar com maior detalhe o sentido da "retomada".

Ce bref article relit le début de la Logique de la philosophie d'Eric Weil et le confronte à la pensée de Hegel. Ce sont ainsi les trois premières catégories de la Logique de la Philosophie qui sont mises en parallèle avec le début de la première partie de la «petite Logique» de Hegel, c'est-à-dire, avec la section Être de l'Encyclopédie des Sciences Philosophiques. Il est intéressant, en effet, de chercher à comprendre ce qui met en mouvement la pensée dialectique, la Vérité chez Weil, l'Etre chez Hegel. La même question se posera dans l'analyse de la seconde catégorie de Weil, le non-sens, dont il s'agira d'interroger la parenté avec le «néant» (das Nichts) de Hegel. C'est alors que surgira le concept de «reprise», dont le contraste avec l'Aufhebung hégélienne apparaîtra de façon surprenante. La catégorie Le vrai et le faux permettra de mieux préciser le sens de la «reprise». 


\section{ÍNDICE}

Palavras-chave: categorias, Hegel, Lógica da Filosofia, retomada, Weil

Mots-clés: catégories, Hegel, Logique de la philosophie, reprise, Weil

\section{AUTOR}

\section{MICHEL RENAUD}

FCSH, Universidade Nova de Lisboa

Belge de naissance, portugais par mariage. Résidant au Portugal depuis 1980. Professeur ordinaire émérite du Département de Philosophie de la Faculté des Sciences Sociales et Humaines de 1 'Université Nouvelle de Lisbonne et professeur ordinaire invité de l'Université Catholique Portugaise. Membre du Conseil National d'Éthique pour les Sciences de la Vie, depuis sa fondation en 1991 et viceprésident du 4 e mandat (2009-2014). Membre effectif de la «Classe de Lettres» de l'Académie des Sciences de Lisbonne. Membre fondateur du Centre d'Études de Bioéthique (Coimbra). Ex-professeur de nomination définitive - de la Faculté des Sciences Philosophiques de l'Université Catholique de Louvain (1973-1980). Conférencier de l'Institut de Défense Nationale (IDN - 1981-2006), des Instituts Supérieurs Militaires et de l'Institut National d'Administration (INA). Publications concernant 1 'anthropologie philosophique, l'herméneutique, l'éthique, la philosophie politique et la philosophie de la religion.

Belga por nascimento, português por casamento. Residindo em Portugal desde 1980. Professor catedrático aposentado do Departamento de Filosofia da Faculdade de Ciências Sociais e Humanas da Universidade Nova de Lisboa e professor catedrático convidado da Universidade Católica Portuguesa. Membro do Conselho Nacional de Ética para as Ciências da Vida desde a sua fundação e vicepresidente do $4^{\circ}$ mandato (2009-2014). Membro efectivo da «Classe de Letras» da Academia das Ciências de Lisboa. Membro fundador do Centro de Estudos de Bioética (Coimbra). Antigo professor de nomeação definitiva - da Faculdade das Ciências Filosóficas de Universidade Católica de Lovaina (Bélgica - 1973-1980). Conferencista do Instituto de Defesa Nacional (IDN - 1981-2006), dos Institutos Superiores Militares e do Instituto Nacional de Administração (INA). Publicações incidindo sobre a antropologia filosófica, a hermenêutica, a ética, a filosofia política e a filosofia da religião. 